\title{
A new method for the calibration of strain cylinders using laser interferometry
}

\author{
Junning Cui ${ }^{1}$, Rolf Kumme ${ }^{2}$, Holger Kahmann ${ }^{2}$ \\ ${ }^{1}$ Harbin Institute of Technology, Harbin, PR China \\ 2 Physikalisch-Technische Bundesanstalt, Braunschweig, Germany
}

\begin{abstract}
This research investigates the possibility of calibrating strain cylinders using laser interferometry, thus providing a new type of transducer that can provide both force and deformation indications. This new method for the calibration of strain cylinders is based on the application of a dual channel laser interferometer in a force standard machine. Using the proposed new method, the relationship between force, deformation, and strain can be calibrated in parallel when calibration forces are applied according to the procedure outlined in ISO 376. Experimental results show that the deformation of a strain cylinder has a definite and stable relationship with the force applied and can be calibrated and directly traced to the wavelength of the laser. Therefore, a new standard that can be used for both alignment verification and indication verification of compression testing machines and other uniaxial testing machines is proven. The research also illustrates the possibility of providing a new deformation-type force transducer using a non-gauged steel cylinder together with a multi-channel laser interferometer.
\end{abstract}

\section{Section: RESEARCH PAPER}

Keywords: strain cylinder; laser interferometry; calibration; deformation; compression testing machine.

Citation: Junning Cui, Rolf Kumme, Holger Kahmann, A new method for the calibration of strain cylinders using laser interferometry, Acta IMEKO, vol. 8, no. 1, article 5, March 2019, identifier: IMEKO-ACTA-08 (2019)-01-05

Editor: Petri Koponen, MIKES Metrology, Finland

Received August 23, 2018; In final form January 31, 2019; Published March 2019

Copyright: (C) 2019 IMEKO. This is an open-access article distributed under the terms of the Creative Commons Attribution 3.0 License, which permits unrestricted use, distribution, and reproduction in any medium, provided the original author and source are credited.

Corresponding author: Rolf Kumme, e-mail: rolf.kumme@ptb.de

\section{INTRODUCTION}

A strain cylinder, also referred to as a strain gauged column, is a type of force transducer with four channel outputs. It is often used for the alignment verification of compression testing machines. Specifications of strain cylinders and the proof procedure for the verification of compression testing machines are specified in international and national standards [1]-[2]. The calibration of strain cylinders can be done in a force standard machine according to ISO standard 376 (ISO 376)[3].

Strain cylinders are used in two main ways. First, since the difference between the signals of the four bridges represent the distribution of the force applied on the strain cylinders, they are used as standards for the alignment verification of compression testing machines, including the self-alignment of upper machine platens, the alignment of component parts of machines, and so on. Second, they can also be used as normal force transducers for force measurement, taking the average signals of the four bridges. Therefore, during the indication calibration of the compression testing machines, strain cylinders can also be used as force transfer standards. Normally, only mechanical qualities like force and strain are of interest during the calibration and application of strain cylinders, while deformation is not given special attention. However, since the platens of the testing machines are sufficiently large and strong, we can ignore integral deformation, and the compression deformation of strain cylinders and local deformation distribution in the surface of platens is mostly decided according to material characteristics. Therefore, in many applications, the deformation is highly significant, and it is often hoped that deformation indication is provided.

Methods and tools in precision engineering have frequently been introduced into the field of force metrology over recent years. For example, laser interferometry has been used in the measurement of the deformation or displacement of key parts [4]-[6], dynamic calibration [7]-[9], and so on. The coordinate measuring technique has been used in on-machine or offmachine measurements of key dimensional parameters [10], [11]. These methods and tools show great capability and advantages in solving certain problems. 


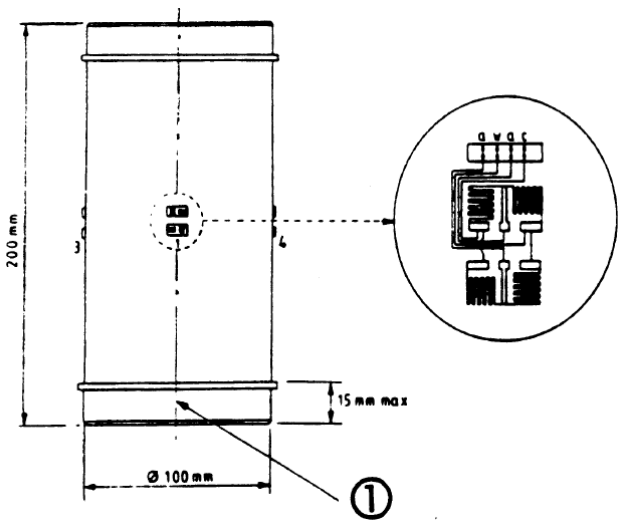

Figure 1. Structure of a strain cylinder and its strain bridges [1].

In this paper, a new method for the calibration of strain cylinders using laser interferometry is proposed and investigated. The new method is based on the application of a dual channel laser interferometer in a force standard machine and shows key benefits and provides important information.

\section{BASIC CONCEPT}

The structures of a strain cylinder and its strain bridges are shown in Figure 1. A strain cylinder consists of a steel cylinder, 16 strain gauges, and other accessories. It has profound demands in terms of its materials, manufacture, assembly, and calibration. The steel cylinder should be precisely manufactured and assembled with 16 matched and temperature-compensated electrical resistance strain gauges. Each four strain gauges are wired in the form of a full bridge, electrically and thermally balanced, and assembled at one of the ends of a pair of orthogonal diameters halfway up the cylinder. Furthermore, the strain cylinder needs to be precisely calibrated on a force standard machine together with the dedicated strain measuring equipment. This calibration qualifies the strain cylinder to be used as a standard for both the alignment verification and indication verification of testing machines.

The conversions of physical quantities during the calibration and application of the strain cylinders are shown in Figure 2. It can be seen that several conversions of physical quantities are involved, during which process the deformation and strain serve as the most important intermediate quantities. At present, strain cylinders are calibrated and used focusing only on the strain rather than the deformation. However, in many applications, the deformation is important and represents key information about alignment, force distribution, and so on. This is the reason why it is often hoped that deformation indication is also provided by strain cylinders. Normally, the platens of compressing testing machines are designed in such a way that they are sufficiently large and strong so as to avoid integral deformation. In turn, the local deformation in the surface of the platens and strain cylinders is only related to their material characteristics. Therefore, important information can be derived from data on deformation.

The aim of the new method for the calibration of strain cylinders is to introduce laser interferometer to a force standard

$\begin{aligned} & \text { Force standard/ } \\ & \text { testing machine }\end{aligned}$
Force
$\longrightarrow$ Conversion of value

Figure 2. Conversions of the physical quantities of strain cylinders. machine and obtain deformation information when calibration forces are applied. It is also hoped that the method will allow for the calibration of the relationship between force, deformation, and strain in parallel. In this way, a new standard that can provide both force and deformation indication is provided, and its use for both the alignment verification and indication verification of compression testing machines is proven. Furthermore, this research illustrates the possibility of providing a new deformation-type force transducer using a non-gauged steel cylinder together with a multi-channel laser interferometer. This new force transducer is much simpler in structure and usage, and it does not require the same complicated manufacturing and assembly processes as strain gauges.

This idea is applicable on the condition that the deformation of a strain cylinder has a definite and stable relationship with the force applied and can be calibrated using a laser interferometer in a force standard machine with good repeatability and reproducibility. This will be proven through experiments and the assessment of this new type of transducer in the following sections.

\section{PERIMENTAL SETUP AND CALIBRATION PROCEDURE}

\subsection{Experimental setup}

The experimental setup is shown in Figure 3. The experiments were carried out in a $5 \mathrm{MN}$ force standard machine. The maximum force applied on the cylinder is $2 \mathrm{MN}$. The strain cylinder, laser heads, and reflectors of the laser interferometer are assembled between the upper and lower platens of the machine, and the strain cylinder is centrally assembled on the lower platen.

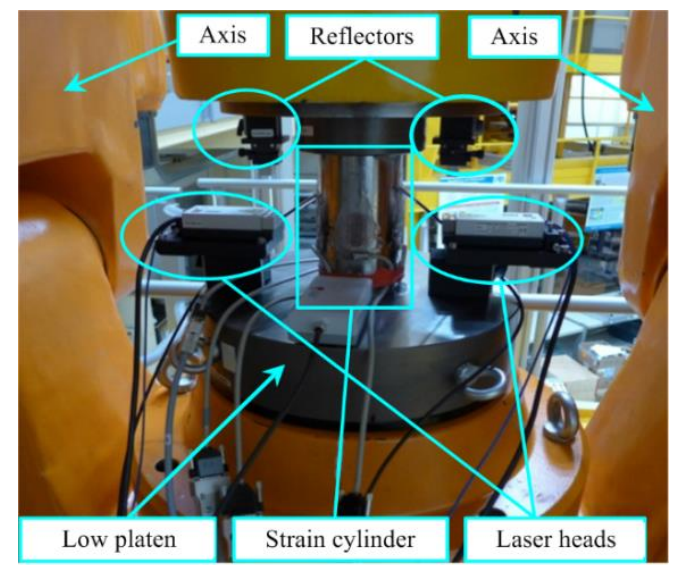

a)

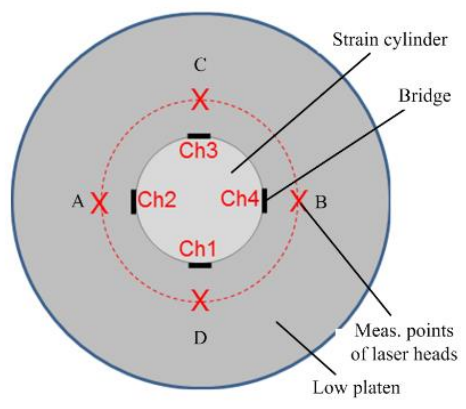

b)

Figure 3. Experimental setup in the $5 \mathrm{MN}$ force standard machine. a) Experimental setup. b) Top view of the strain cylinder with four bridges and the four corresponding measurement points. 
The strain cylinder is $100 \mathrm{~mm}$ in diameter and $200 \mathrm{~mm}$ in height. It conforms to the requirements for material and dimensional tolerances in [1]. Four full bridges of strain gauges, labelled Channels 1 to 4, are assembled at fours ends of a pair of orthogonal diameters halfway up the cylinder. Each bridge consists of two elements measuring the axial strain and two elements measuring the circumferential strain. The outputs of the four bridges are recorded by the dedicated strain measuring equipment DMP41.

A MI-5000 dual channel laser interferometer from SIOS Meßtechnik $\mathrm{GmbH}$ is used. Its relative length measurement accuracy is up to $1 \times 10^{-6}$; therefore, the length measurement error introduced during the experiments is negligible, showing the great advantage of this precision engineering tool. For each channel, interferometric optics, excluding the measuring reflector, are assembled together as a laser head. The laser is transmitted to each of the two laser heads through a length of optical fibre. Corresponding to the orientations of the four bridges on the strain cylinder, there are four measurement points for the laser heads on the lower platen, which are at four ends of a pair of corresponding orthogonal diameters of a circle and designated as A, B, C, and D in Figure 3 b). In such a way, the strain cylinder together with the laser heads work as a new type of transducer with four 'deformation sensing elements'. The four 'deformation sensing elements' are uniformly distributed over $360^{\circ}$ and labelled as Channels 1 to 4 respectively.

The distance between the measurement points and the sidewall of the strain cylinder should be kept as small as possible. In this study, it is $60 \mathrm{~mm}$. The influence of this distance and the resultant deformation distribution in the surface of the platens can be taken into consideration in two ways: 1 ) when the strain cylinder is used in a compression testing machine, the same parameter should be maintained. Usually, the platens of the compression testing machine and the force standard machine are made of material similar to Young's elastic modulus. The error introduced should be corrected if there is an obvious difference; 2) the deformation to the central position of the platens for both the force standard machine and the compression testing machine should be corrected.

\subsection{Calibration procedure}

The procedure for calibrating a strain cylinder using a dual channel laser interferometer is proposed with reference to ISO 376. The cylinder is rotated around its axis to four uniformly distributed positions over $360^{\circ}$, as shown in Figure 4. Deformation and strain data at the four rotation positions are averaged to eliminate the influence of the non-uniform force distribution characteristics of the force standard machine. A series of increasing calibration forces and then a series of decreasing calibration forces are applied at each rotation position. Because strain cylinders are only used for compression testing, the data corresponding to the decreasing calibration forces are not used for interpolation, but are only used for the assessment of the relative reversibility error in Section 4.3. The two laser heads are first assembled at Point A and Point B, and the strain cylinder is calibrated at the four rotation positions. Then, the two laser heads are reassembled to Point $\mathrm{C}$ and Point $\mathrm{D}$, and the strain cylinder is calibrated at another four rotation positions. In such a way, the outputs of the four channel 'deformation sensing elements' of the new type of transducer can be obtained at each rotation position.

The series of increasing calibration forces are set from 0 to $2000 \mathrm{kN}$, with a $200 \mathrm{kN}$ increment, and the series of decreasing

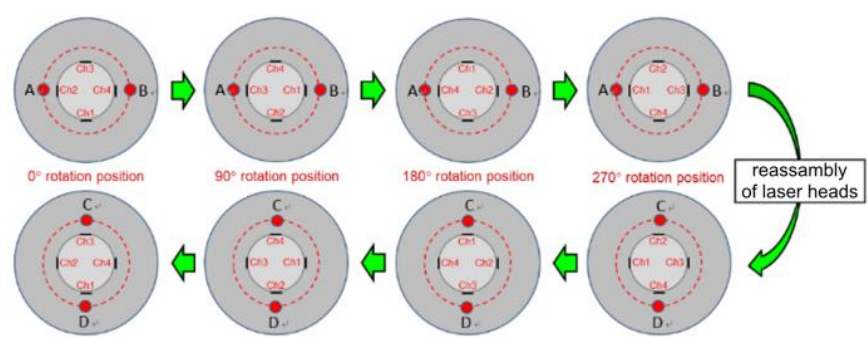

Figure 4. The procedure for calibrating a strain cylinder using a dual channel laser interferometer.

calibration forces are set from $2000 \mathrm{kN}$ down to $0 \mathrm{kN}$, with a $200 \mathrm{kN}$ decrement. In total, 11 calibration forces (including zero force), uniformly distributed over the calibration range, are applied. There is a wait of $\sim 15 \mathrm{~s}$ after a calibration force is applied, waiting for the force to become stable. Then, the calibration force is maintained, unchanged, for $\sim 45 \mathrm{~s}$ before it is changed to the next successive value.

The data recorded by DMP41 is designated as $X_{c b i, j}$ and $X_{c b i, j}{ }^{\prime}$ in the following sections, where $i$ is the channel number of strain bridges $(i=1,2,3$, or 4$), j$ is the rotation position of the strain cylinder $(j=0,90,180$, or 240$), X_{c b i, j}$ corresponds to the increasing calibration forces, and $X_{c h i, j}{ }^{\prime}$ corresponds to the decreasing calibration forces. Similarly, the data recorded by the laser interferometer is designated as $Y_{c h i, j}$ and $Y_{c b i, j}{ }^{\prime}$, where $i$ is the channel number of 'deformation sensing elements'.

\subsection{Data sampling setting}

It is a good idea to use a high data sampling rate based on the strategy of 'more data, more information'. However, a high sampling rate results in a high demand for hardware and software, making the data processing difficult. On the other hand, according to general knowledge concerning the force standard machine, the natural frequency of the micro-vibration of the lower platen is $\sim 0.27 \mathrm{~Hz}$, so the period of vibration is $\sim 3.7$ $\mathrm{s}$. Therefore, the data sampling rates of the laser interferometer and DMP41 are both set at $10 \mathrm{~Hz}$. Therefore, the sampling can be regarded as 'dynamic' compared to the period of platen vibration, thereby including any important information.

\section{DATA PROCESSING AND EXPERIMENTAL RESULTS}

\subsection{Data pre-processing}

The abundance of data continuously sampled, at a sampling rate of $10 \mathrm{~Hz}$, are pre-processed first so as to obtain the values of deformation and strain corresponding to the 11 calibration forces during each incremental force loading and decremental force loading.

For the data on the laser interferometer during each calibration, first, the corner points corresponding to the moments when a calibration force begins to increase or decrease to the next successive value are determined with a slope-judging algorithm, marked with a green $*$ in Figure 5 . Then, the average values of the $25 \mathrm{~s}$ data before each corner point are calculated, marked with green lines in Figure 5. This averaging operation works as a low-pass filter (LPF) with a cut-off frequency of $0.04 \mathrm{~Hz}$, thus eliminating the influence of the resultant noise from the mechanical vibration and other factors. The deformation values corresponding to 10 calibration forces during each incremental force loading and decremental force loading are obtained. 


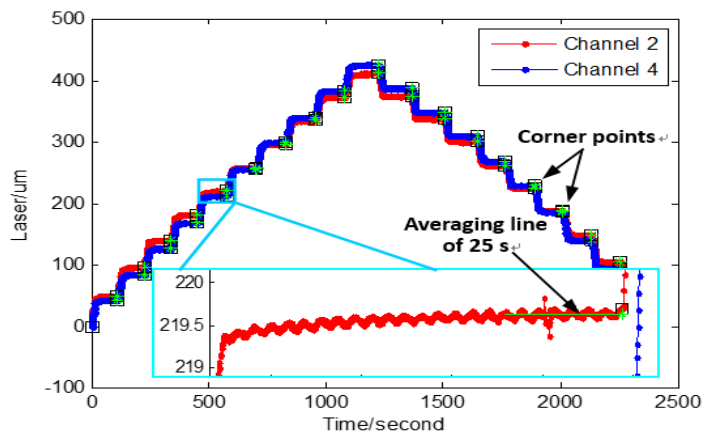

Figure 5. Pre-processing of the data on the laser interferometer $\left(0^{\circ}\right.$ rotation position).

Theoretically, the indication of the laser interferometer should be reset to zero at the moment at which the upper platen comes into contact with the strain cylinder i.e. the moment at which the calibration force begins to increase from zero. It is a good solution to send a trigger signal to the laser interferometer either automatically or manually, or to use one of the following two methods: (1) during data pre-processing, the data corresponding to zero force should be recognised and all successive data should be subtracted by the corresponding zero data, which is the case in this research; (2) the values of deformation corresponding to 10 calibration forces from $200 \mathrm{kN}$ to $2000 \mathrm{kN}$ should be used for interpolation, and the deformation value from 0 to $200 \mathrm{kN}$ through extrapolation should be calculated. All deformation values from 0 to $2000 \mathrm{kN}$ should be subtracted by the value corresponding to zero force.

The data on DMP41 is similarly pre-processed, as shown in Figure 6. The only exception is that the strain value corresponding to zero force can be directly calculated by averaging the $25 \mathrm{~s}$ data before the corresponding corner point. In total, 11 strain values corresponding to the 11 calibration forces (including zero force) are used for interpolation.

\subsection{Data processing results}

By means of data pre-processing, the four channels' values of deformation and strain corresponding to the 11 calibration forces at each rotation position, either increasing or decreasing, are obtained. The interpolation curves are determined using the average values of deformation and strain at the four rotation positions. As mentioned above, data corresponding to the decreasing calibration forces is not used for interpolation. Threedegree polynomial fitting is carried out to obtain third-degree equations about the relationship between force, deformation, and strain.

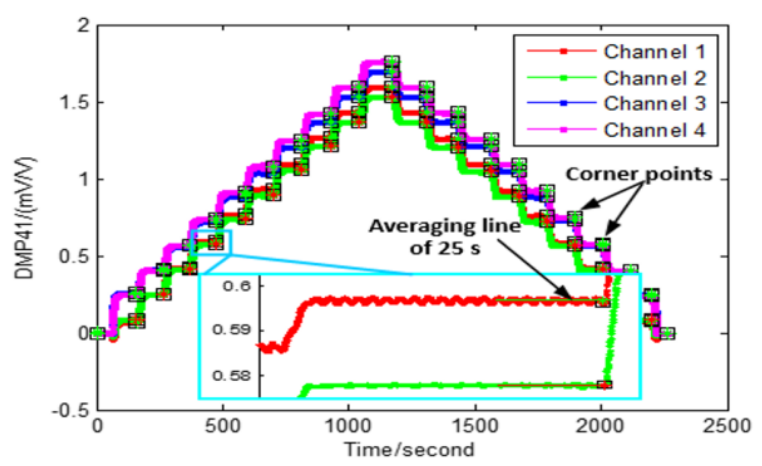

Figure 6. Pre-processing of the data on the four channels of DMP41 $\left(0^{\circ}\right.$ rotation position).

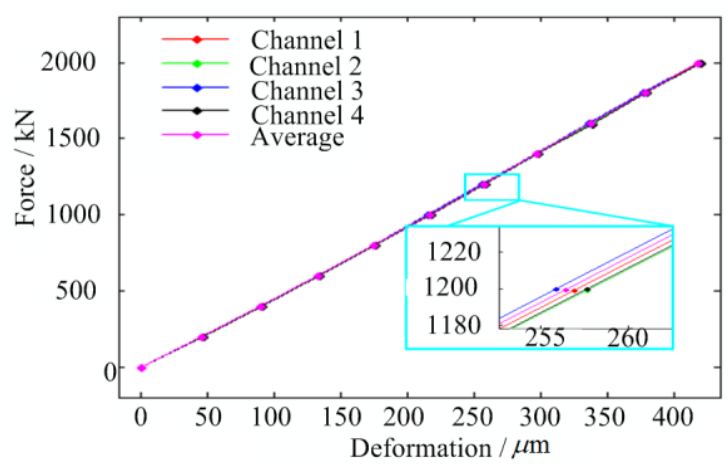

Figure 7. Curves showing the relationship between force and deformation.

\subsubsection{The polynomial fitting results of force vs. deformation}

The equations for calculating the deformation as a function of strain are as follows:

$$
\begin{gathered}
\left\{\begin{array}{l}
F=-2.013 \cdot 10^{-6} \cdot Y_{c h 1}{ }^{3}+0.002064 \cdot Y_{c h 1}{ }^{2}+4.272 \cdot Y_{c h 1}-0.4283 \\
F=-1.748 \cdot 10^{-6} \cdot Y_{c h 2}{ }^{3}+0.001859 \cdot Y_{c h 2}{ }^{2}+4.337 \cdot Y_{c h 2}+0.3774 \\
F=-2.263 \cdot 10^{-6} \cdot Y_{c h 3}{ }^{3}+0.002216 \cdot Y_{c h 3}{ }^{2}+4.270 \cdot Y_{c h 3}+0.06657 \\
F=-2.356 \cdot 10^{-6} \cdot Y_{c h 4}{ }^{3}+0.002293 \cdot Y_{c h 4}{ }^{2}+4.226 \cdot Y_{c h 4}-0.7880
\end{array}\right. \\
F=-2.096 \cdot 10^{-6} \cdot \bar{Y}^{3}+0.0021083 \cdot \bar{Y}^{2}+4.276 \cdot \bar{Y}-0.2008
\end{gathered}
$$

where $F$ is the calibration force in $\mathrm{kN} ; Y_{c b i}$ is the deformation of Channel $i$ in $\mu \mathrm{m} ; i=1,2,3$, or 4 ; and $\bar{Y}$ is the average deformation of the four channels.

The curves showing the four third-degree equations are shown in Figure 7. It can be seen that the four channels of the strain cylinder have very good consistency.

It can be concluded that the deformation of a strain cylinder has a definite and stable relationship with the force applied by the force standard machine. Equation (1) and Equation (2) also illustrate the possibility of providing a new deformation-type force transducer using a non-gauged steel cylinder together with a multi-channel laser interferometer, using either average or nonaveraged values of the deformation of the four channels. The average value represents the amplitude of the force applied on the strain cylinder, while the non-averaged values of the four channels illustrate the force distribution.

\subsubsection{The polynomial fitting results of deformation vs. strain and force vs. strain}

The equations for calculating the deformation as a function of strain are as follows:

$$
\left\{\begin{array}{l}
Y_{c h 1}=8.584 \cdot X_{c h 1}{ }^{3}-31.77 \cdot X_{c h 1}{ }^{2}+283.3 \cdot X_{c h 1}+0.2128 \\
Y_{c h 2}=3.058 \cdot X_{c h 2}{ }^{3}-15.29 \cdot X_{c h 2}{ }^{2}+268.0 \cdot X_{c h 2}-0.5468 \\
Y_{c h 3}=7.588 \cdot X_{c h 3}{ }^{3}-28.62 \cdot X_{c h 3}{ }^{2}+279.4 \cdot X_{c h 3}+0.03244 \\
Y_{c h 4}=13.35 \cdot X_{c h 4}{ }^{3}-45.43 \cdot X_{c h 4}{ }^{2}+294.3 \cdot X_{c h 4}+1.062
\end{array}\right.
$$

$\bar{Y}=8.102 \cdot \bar{X}^{3}-30.22 \cdot \bar{X}^{2}+281.3 \cdot \bar{X}+0.1592$

where $X_{\mathrm{ch} i}$ is the strain indication of Channel $i$ in $\mathrm{mV} / \mathrm{V} ; i=1$, 2,3 , or 4 ; and $\bar{X}$ is the average strain of the four channels. 
The curves showing the four third-degree equations are shown in Figure 8. Since the four strain bridges are well matched as well as thermally and electrically balanced, so too do these four curves have good consistency.

Equations giving the calibration force as a function of strain can be similarly calculated, as follows:

$$
\begin{aligned}
& \left\{\begin{array}{l}
F=3.942 \cdot X_{c h 1}{ }^{3}-9.654 \cdot X_{c h 1}{ }^{2}+1221 \cdot X_{c h 1}+0.04842 \\
F=-15.24 \cdot X_{c h 2}{ }^{3}+48.81 \cdot X_{c h 2}{ }^{2}+1168 \cdot X_{c h 2}-2.339 \\
F=-3.916 \cdot X_{c h 3}{ }^{3}+12.59 \cdot X_{c h 3}{ }^{2}+1204 \cdot X_{c h 3}-0.2183 \\
F=19.09 \cdot X_{c h 4}{ }^{3}-55.75 \cdot X_{c h 4}{ }^{2}+1261 \cdot X_{c h 4}+3.191
\end{array}\right. \\
& F=0.8159 \cdot \bar{X}^{3}-0.7826 \cdot \bar{X}^{2}+1214 \cdot \bar{X}+0.06329
\end{aligned}
$$

During real applications, either the average signal or the nonaveraged signals of the four channel strain bridges can be used. The average signal represents the amplitude of the force applied on the strain cylinder, while the non-averaged values of the four channels illustrates the force distribution applied on the strain cylinder.

Using Equation (3), Equation (4), Equation (5), and Equation (6), a new type of transducer that provides both force indication and deformation indications is provided. This new type of transducer can be used as a standard for both the alignment verification and indication verification of compression testing machines.

\subsection{Assessment of the deformation calibration results}

The calibration results of deformation $Y$ are assessed with reference to ISO 376. The relative length measurement accuracy of the MI-5000 laser interferometer is up to $1 \times 10^{-6}$, and the uncertainty of the calibration forces applied by the $5 \mathrm{MN}$ force standard machine is up to $1 \times 10^{-4}(k=2)$; therefore, this assessment can be used to verify the proposed new method. Data corresponding to decremental force loading is used during the assessment of relative reversibility error.

\subsubsection{Relative reproducibility error}

The relative reproducibility error of deformation value $Y$ assesses the variation of the deformation indication at different rotation positions. The average deformation of the four channels is used for assessment. The error is calculated using the following equations in accordance with ISO 376:

$\left\{\begin{array}{l}b=\left|\frac{\max \bar{Y}-\min \bar{Y}}{\bar{Y}_{\text {ave }}}\right| \times 100 \% \\ \bar{Y}_{\text {ave }}=\frac{\bar{Y}_{0}+\bar{Y}_{90}+\bar{Y}_{180}+\bar{Y}_{270}}{4}\end{array}\right.$

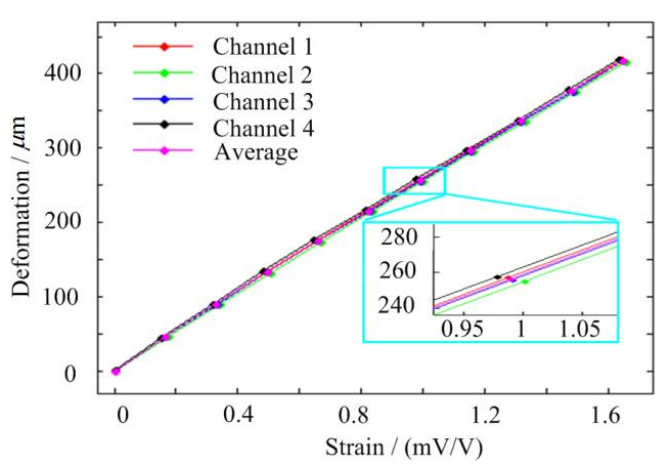

Figure 8. Curves showing the relationship between deformation and strain.

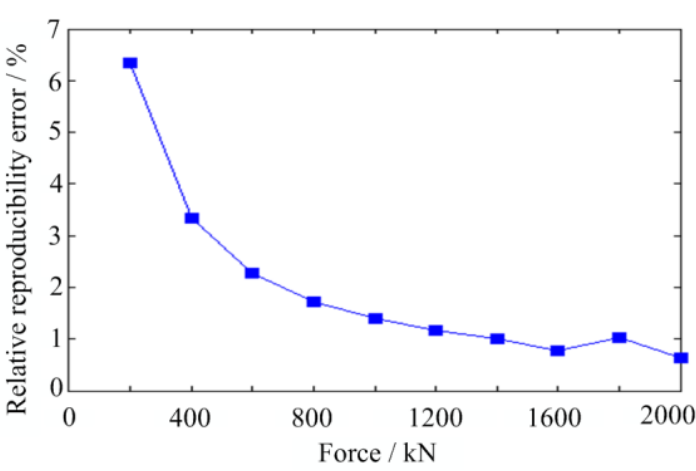

Figure 9. Relative reproducibility errors in \%.

The relative reproducibility errors are shown in Figure 9. The maximum relative reproducibility error $b_{\max }$ results when the calibration force is $200 \mathrm{kN}$ and $b_{\max }=6.4 \%$. We found that the reproducibility error mostly results due to the non-uniform force distribution characteristics of the force standard machine. The indication variation of deformation at different rotation positions and the relative reproducibility error can be significantly reduced if the force distribution is more uniform.

\subsubsection{Relative interpolation error}

The relative interpolation error is calculated for each channel using the following equation:

$$
f c_{c h i}=\frac{\bar{Y}_{c h i}-Y a_{c h i}}{Y a_{c h i}} \times 100 \%
$$

where $Y a_{c b i}$ is the value of deformation of Channel $i$ computed by means of inverse functions of Equation (1). These functions give the deformation as a function of the calibration force.

The relative interpolation errors of Channels 1 to 4 are shown in Figure 10. The maximum relative interpolation error $\left|f_{c}\right|_{\text {max }}$ results in Channel 4 when the calibration force is $200 \mathrm{kN},|f c|_{\text {max }}$ $=0.84 \%$. The relative interpolation error can be averaged if the average deformation of the four channels is used.

\subsubsection{Relative zero error}

The relative zero error of Channel $i$ at $j$ degree rotation position is calculated using the following equation:

$$
f o_{c h i, j}=\frac{Y f_{c h i, j}-Y o_{c h i, j}}{Y N_{c h i, j}} \times 100 \%
$$

where $Y_{c b i}$ is the deformation taken before the calibration force is applied, and $Y f_{c h i, j}$ is the deformation taken after the calibration

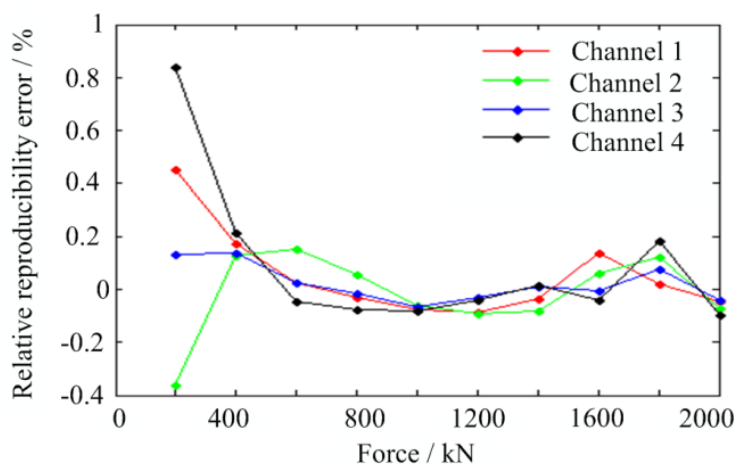

Figure 10. Relative interpolation errors of the four channels. 


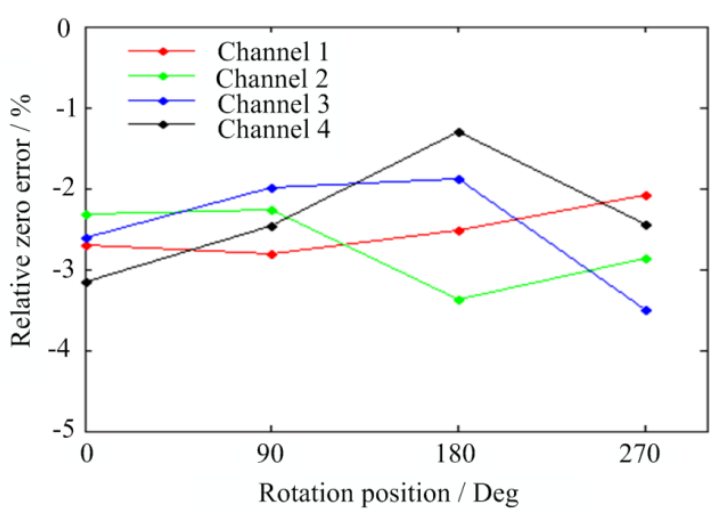

Figure 11. Relative zero errors of the four channels.

force is completely removed. $Y N_{c h i j}$ is the deformation corresponding to the $2000 \mathrm{kN}$ maximum calibration force.

The relative zero errors of Channels 1 to 4 are shown in Figure 11. The maximum relative zero error $f_{\text {max }}$ results in Channel 3 when the cylinder is set at a $270^{\circ}$ rotation position and $f_{\max }=-3.5 \%$.

\subsubsection{Relative reversibility error}

The relative reversibility error of Channel $i$ at $j$ degree rotation position $v_{c b i j}$ and the relative reversibility error of Channel $i v_{c b i}$ are calculated using the following equations respectively:

$$
\left\{\begin{array}{l}
v_{c h i, j}=\left|\frac{Y_{c h i, j}{ }^{\prime}-Y_{c h i, j}}{Y_{c h i, j}}\right| \times 100 \% \\
v_{c h i}=\frac{v_{c h i, 0^{\circ}}+v_{c h i, 90^{\circ}}+v_{c h i, 180^{\circ}}+v_{c h i, 270^{\circ}}}{4}
\end{array}\right.
$$

The relative reversibility errors of Channels 1 to 4 are shown in Figure 12. The maximum relative reversibility error $v_{\max }$ results in Channel 4 when the calibration force is $200 \mathrm{kN}$ and $v_{\max }=$ $13 \%$. The reversibility error mostly results from the hysteresis between the incremental loading and the decremental force loading. Since strain cylinders are only used for compression testing, this is unproblematic.

\subsubsection{Relative creep error}

The relative creep error of Channel $i$ is calculated using the following equation:

$$
c_{c h i}=\frac{Y 200_{c h i}-Y 20_{c h i}}{Y N_{c h i}} \times 100 \%
$$

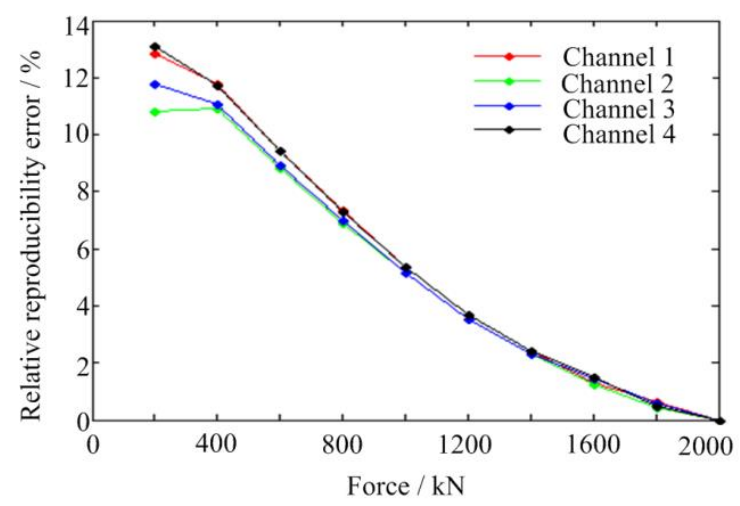

Figure 12. Relative reversibility errors of the four channels.

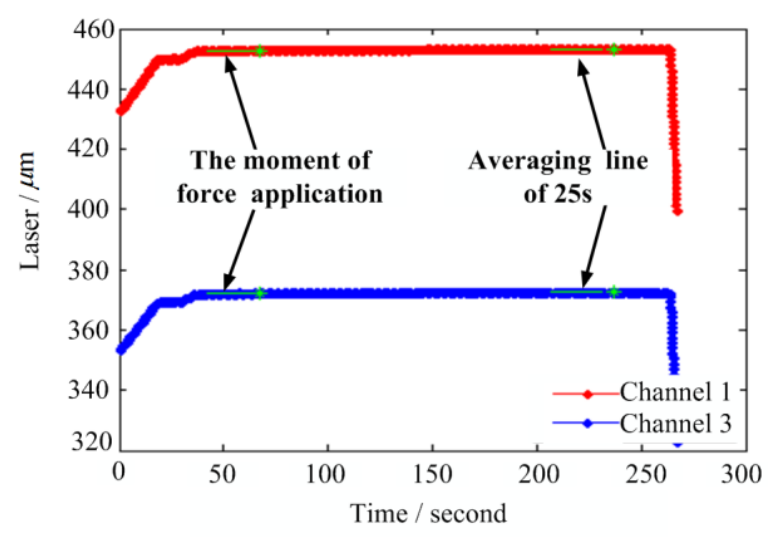

Figure 13. Experimental results of the relative creep error test.

where $Y 20_{c b i}$ and $Y 200_{c b i}$ are deformation indications obtained $20 \mathrm{~s}$ and $200 \mathrm{~s}$ after the maximum calibration force is applied; and $Y N_{c b i}$ is the maximum deformation corresponding to the $2000 \mathrm{kN}$ maximum calibration force. Remark: According to ISO 376 , the creep should be measured within $300 \mathrm{~s}$, but in this experiment, the creep was measured within $200 \mathrm{~s}$ in order to reduce the measurement time.

The calculation of the average of the $25 \mathrm{~s}$ data that works as a $0.04 \mathrm{~Hz} \mathrm{LPF}$ is used. The experimental results of the relative creep error test are shown in Figure 13. The maximum relative creep error $c_{\max }=0.091 \%$.

\subsection{Analysis of influential factors}

Several main influential factors concerning the experimental results can be analysed. This analysis should reveal the value of the influence of the force cylinder tolerance and how the influence of the non-ideal characteristics of the force standard machine [12]-[15] is greatly reduced by means of the calibration procedure design proposed in ISO 376.

\subsubsection{Influence of the rotation of the strain cylinder}

According to the experimental procedure design, the influence of the rotation of the strain cylinder can be evaluated by means of the reproducibility of a single laser head assembled at a fixed position, while the strain cylinder is calibrated at four rotation positions.

As shown in Figure 14, the measurement results of the laser head fixed at Point $\mathrm{C}$ have very good reproducibility when incremental forces are applied on the strain cylinder at the four rotation positions. This proves that the rotation of a force cylinder, which conforms to the requirements of material and dimensional tolerance, has nearly no influence on the measurement results. We can ignore the non-parallelism of its upper and lower surfaces and other dimensional tolerance, unlike the dimensional tolerance of the force standard machines.

\subsubsection{Averaging the different rotation positions}

The method of averaging measurement results at multiple rotation positions is proposed in ISO 376. Theoretically, the influence of non-uniform force distribution, contact effect, and so on can be significantly reduced using this method. In this study, the difference between the characteristics of the different channel strain bridges in the strain cylinder is less than $0.1 \%$. Therefore, the influence of the averaging of the different rotation positions can be evaluated by means of a comparison between the different channel calibration results. 


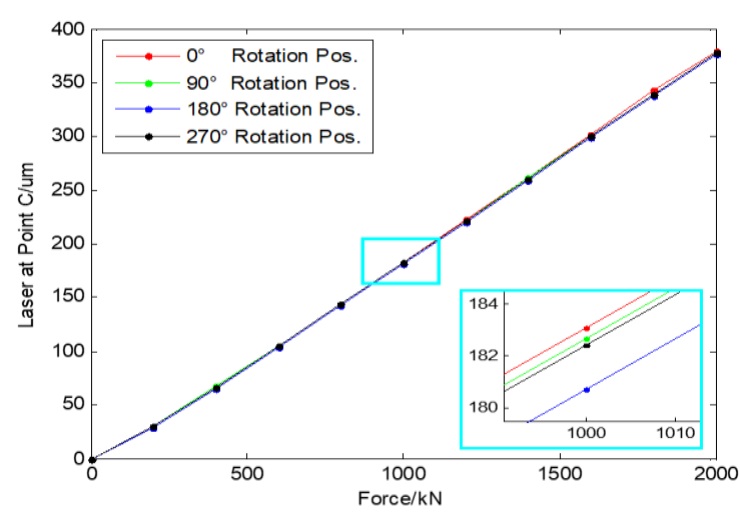

Figure 14. Deformation at Point $C$ when incremental force is applied the four rotation positions.

The calibration results of the four-channel relationship between strain and force are shown in Figure 15. The maximum deviation of strain when $200 \mathrm{kN}$ force is applied is $0.007 \mathrm{mV} / \mathrm{V}$. The maximum strain when the $2000 \mathrm{kN}$ maximum force is applied is $1.6445 \mathrm{mV} / \mathrm{V}$; therefore, the relative difference is $((0.007 \mathrm{mV} / \mathrm{V}) /(1.6445 \mathrm{mV} / \mathrm{V})) \times 100 \%=0.4 \%$. It is proven that the error introduced by non-uniform force distribution characteristics, contact effect, and so on are effectively reduced by averaging the different rotation positions.

\subsection{Proposals}

In this research, the calibration procedure, data processing method, and assessment of the transducer calibration are designed with reference to ISO 376. However, some suggestions and proposals should be noted:

(1) The cylinder should be rotated symmetrically around its axis to at least three uniformly distributed positions over $360^{\circ}$, thus eliminating the influence of the non-uniform force distribution characteristics of the force standard machine;

(2) In this research, the incremental and decremental force loading are both applied on the strain cylinder. Since strain cylinders are only used for compression testing, the data corresponding to decremental force loading is only used for assessment rather than interpolation. In order to get as much information as possible, 'dynamic' data sampling is advisable, but not mandatory;

(3) During data pre-processing, the calculation of deformation and strain corresponding to zero force should be given special attention. Readings of strain bridges can be directly

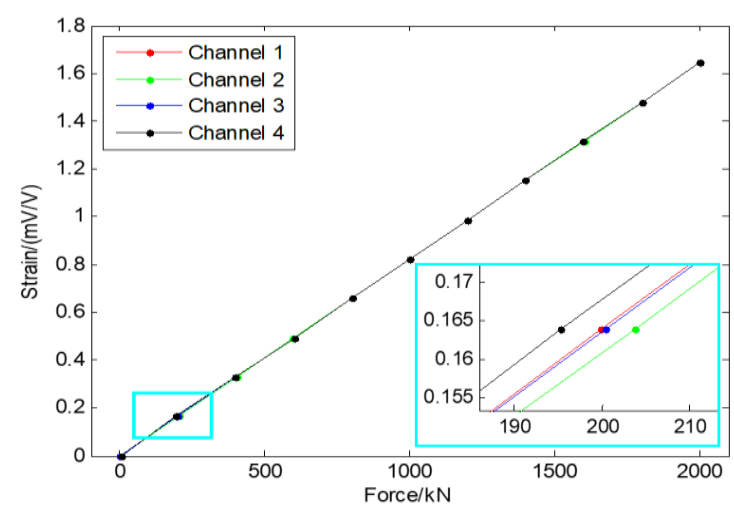

Figure 15. The calibration results of the four-channel relationship between strain and force. taken before the force is applied or after the force has been completely removed. However, readings of the laser interferometer corresponding to zero force should be taken at the moment at which the upper platen comes into contact or loose contact with the cylinder. The indication of the laser interferometer can be reset to zero at this moment, using the methods recommended in Section 4.1; and

(4) If a non-gauged steel cylinder is calibrated using the proposed method and used together with a multi-channel laser interferometer, a 'deformation-type' force transducer is provided. The distance between measurement laser beams and the sidewall of the cylinder should be kept as small as possible, and the error introduced can be considered using the methods recommended in Section 3.1. If the deformation of platens is negligible because the calibration force is too small or the platens are sufficiently strong, this does not prove problematic.

\section{CONCLUSION}

A new method for the calibration of strain cylinders using laser interferometry was proposed and investigated in this study. This new method was illustrated by means of calibrating a strain cylinder with a SIOS dual channel laser interferometer in a $5 \mathrm{MN}$ force standard machine at PTB. The calibration procedure, data processing method, and assessment of calibration results were designed with reference to ISO 376. Experimental results show that the deformation of a strain cylinder has a definite and stable relationship with the force applied and can be calibrated and directly traced to the wavelength of laser.

Therefore, a new type of transducer that can provide both force and deformation indications has been proven herein, and it can be used for both the alignment verification and indication verification of compression testing machines and other uniaxial testing machines. This method can also be applied to non-gauged standard cylinders, thus providing a new deformation-type force transducer using a non-gauged steel cylinder together with a multi-channel laser interferometer.

\section{ACKNOWLEDGEMENT}

The authors gratefully acknowledge the valuable help of Heiko Wunderlich of PTB on the experimental operation, and a constructive technical discussion with Michael Wagner of PTB.

\section{REFERENCES}

[1] EN 12390-4: Testing hardened concrete - Part 4: Compressive strength - Specification for testing machines, 2000.

[2] DIN 51302-2: Strain cylinder test for verifying compression testing machines for testing concrete, 2000.

[3] ISO 376: Metallic materials - Calibration of force-proving instruments used for the verification of uniaxial testing machines, 2011.

[4] C. Junning, R. Kumme, H. Kahmann, Proposal and investigation of a new method for calibration of strain cylinders using laser interferometry, Proc. of the $23^{\text {rd }}$ IMEKO TC3 Conference on the Measurement of Force, Mass and Torque, Helsinki, Finland, 30 May - 01 June 2017.

[5] Gordon A. Shaw, Koo-Hyun Chung, Douglas T. Smith et al., Methods for transferring the SI unit of force from millinewtons to piconewtons. Proceedings of the SEM Annual Conference, Albuquerque New Mexico USA. 1 - 4 June 2009.

[6] C. Bartoli, M. F. Beug, T. Bruns et al., Traceable dynamic measurement of mechanical quantities: objectives and first results of this European project, Int. J. Metrol. Qual. Eng. 3 (2012), pp. 127-135. 
[7] L. Zhang, R. Kumme, Investigation of interferometric methods for dynamic force measurement, Proc. of the XVII IMEKO World Congress, Dubrovnik, Croatia, 22 - 28 June 2003, pp. 315318

https://www.imeko.org/publications/wc-2003/PWC-2003TC3-015.pdf.

[8] Y. Fujii, J. D. R. Valera, Impact force measurement using an inertial mass and a digitizer, Meas. Sci. Technol. 17 (2006), pp. 863 868.

[9] Y. Fujii, Optical method for accurate force measurement: dynamic response evaluation of an impact hammer. Optical Engineering 45, 2 (2006), 023002-1-7.

[10] S. Baumgarten, D. Röske, H. Kahmann et al., Concept and setup of a multi-component facility for force and torque in the range of $1 \mathrm{MN}$ and $2 \mathrm{kN} \cdot \mathrm{m}$, ACTA IMEKO 3 (2014) 2, pp. 39-43, DOI: https://doi.org/10.21014/acta imeko.v3i2.66.
[11] D. Röske, K. Adolf, D. Peschel, Lever optimization for torque standard machines, Proc. of the XVI IMEKO World Congress, Vienna, Austria, 25 - 28 September 2000, https://www.imeko.org/publications/wc-2000/IMEKO-WC2000-TC3-P100.pdf.

[12] Y.-K. Park, D.-I. Kang, Oscillating signal components of a deadweight force-standard machine and reduction techniques, Meas. Sci. Technol. 10 (1999), pp. 748-754.

[13] Y.-K. Park, D.-I. Kang, Pendulum motion of a deadweight forcestandard machine, Meas. Sci. Technol. 11 (2000), pp. 1766-1771.

[14] S. K. Jain, H. Kumar, S. S. K. Titus, et al., Metrological characterization of the new $1 \mathrm{MN}$ force standard machine of NPL India, Measurement 45, 3 (2012), pp. 590-592.

[15] T. Hayashi, Y. Katase, H. Maejima, Recent renovations of deadweight type force standard machines at NMIJ, Measurement 46, 10 (2013), pp. 2127-2134. 